ISSN: 1130-3743 - eISSN: 2386-5660

DOI: http://dx.doi.org/10.14201/teoredu302722

\title{
EL PENSAMIENTO CRÍTICO EN CRISIS. UNA RECONSIDERACIÓN PEDAGÓGICA EN TRES MOVIMIENTOS
}

\section{Critical Thinking in Crisis. A Pedagogical Reconsideration in Three Movements}

\section{La Pensée Critique en Crise. Une Reconsidération Pédagogique en Trois Mouvements}

\author{
Paul STANDisH* y Bianca THOILLIEZ** \\ *University College of London. Institute of Education, 20 Bedford Way, \\ Bloomsbury, London WC1H OAL, Reino Unido \\ * Universidad Autónoma de Madrid. Facultad de Formación de Profesorado y \\ Educación. Departamento de Didáctica y Teoría de la Educación. Campus de \\ Cantoblanco. C/ Francisco Tomás y Valiente. 28049 Madrid. \\ p.standish@ucl.ac.uk; bianca.thoilliez@uam.es
}

Fecha de recepción: enero de 2018

Fecha de aceptación: marzo de 2018

\section{RESUMEN}

El objetivo de este artículo es poner en crisis el tratamiento dominante que recibe la actividad del pensamiento crítico en el ámbito educativo. En concreto, se van a proponer una serie de tres variaciones en el modo de considerar el elemento "crisis» dentro de la conceptualización y práctica del pensamiento crítico. En primer lugar, se propone ofrecer una imagen más real del ser humano, para quien sus dudas e incertidumbres continuas están permanentemente entreveradas y mezcladas. En segundo lugar, se plantea revalorizar la función de la imaginación mostrando su dificultad para ser enseñada a través de una serie de pasos preestablecidos. En tercer 
lugar, se sugiere valorar más el modo en que los problemas se elaboran, y no solo la perspectiva de cómo llegamos a solucionarlos. El artículo termina proponiendo que la mejor vía para desarrollar un pensamiento más auténticamente crítico es la transmisión de un contenido disciplinar rico en ideas e inserto en una tradición sujeta al diálogo y el debate continuos.

Palabras clave: pensamiento crítico; sentido; contenidos de enseñanza; tradición; lectura.

\section{SUMMARY}

The objective of this article is to put in crisis the dominant approach that critical thinking receives in the field of education. In particular, a series of three variations will be proposed in the way of considering the element of "crisis» within the conceptualization and practice of critical thinking. Firstly, a more real image of the human being is proposed, for whom his doubts and continuous uncertainties are permanently interwoven and mixed. Secondly, the function of the imagination is proposed to be revalued, showing how difficult is to teach it through a series of pre-established steps. Thirdly, it is suggested more value should be granted to the way in which problems are elaborated, and not focus exclusively on how we come to solve them. The article ends by proposing that the best way to develop a more authentically critical thinking is the transmission of disciplinary contents rich in ideas and inserted in a tradition subject to continuous dialogue and debate.

Key words: critical thinking; meaning; teaching contents; tradition, reading.

\section{SOMMAIRE}

L'objectif de cet article est de mettre en crise le traitement dominant qui reçoit l'activité de pensée critique dans le domaine de l'éducation. En particulier, une série de trois variantes sera proposée à fin de reconsidérer l'élément "crise» dans la conceptualisation et la pratique de la pensée critique. Premièrement, il est proposé d'offrir une image plus réelle de l'être humain, pour qui ses doutes et ses incertitudes continues sont entremêlés et mélangés en permanence. Deuxièmement, il est proposé de réévaluer la fonction de l'imagination en montrant sa difficulté à être enseigné à travers une série d'étapes préétablies. Troisièmement, il est suggéré de valoriser davantage la manière dont les problèmes sont élaborés, et non seulement se concentrer sur la manière dont nous arrivons à les résoudre. L'article conclut en proposant que la meilleure façon de développer une pensée plus authentiquement critique est la transmission d'un contenu riche en idées et inséré dans une tradition portée à un dialogue et un débat continus.

Mots clés: pensée critique; sens; contenus d'enseignement; tradition, lecture. 


\section{INTRODUCCIÓN}

El encuentro con la realidad nos sume en una cierta crisis y desequilibrio. Para combatir esa misma crisis nos vemos movidos a hacerlo críticamente. Además, nuestro encuentro con la realidad lo hacemos siempre en el contexto de una determinada tradición. Al nacer lo hacemos en un mundo desconocido en el cual debemos ser iniciados a modo de "segundo nacimiento" (Bárcena, 2006), siendo la tradición heredada aquello en lo que nos vemos introducidos. De hecho, la misma actividad de pensar críticamente forma parte de los legados pedagógico y filosófico que hemos recibido. Por un lado, el énfasis que en la educación damos al pensamiento crítico concuerda con la profunda estructura racionalista de nuestra tradición pedagógica occidental. Por otro lado, la historia intelectual de Occidente ha sido abordada desde sus inicios como una tarea eminentemente crítica. Cuando, desde la modernidad cartesiana, el "yo» que piensa es elevado a criterio de discernimiento acerca de sí mismo y de lo que piensa, y cuando la realidad es entonces una representación sujeta a interpretación, la experiencia del encuentro con la realidad se torna necesaria y eminentemente "crítica". La situación cultural actual nos ha llevado a un punto en el que "la tensión entre aquello que pretende "ejercer crítica" y aquello que sería criticable es tan fuerte que nuestro pensamiento se hace cien veces más hosco que preciso [...] Dado que todo se hizo problemático, también todo, de alguna manera, da lo mismo» (Sloterdijk, 2007, 21). Una situación que hace del pensar una actividad más vacilante y dubitativa y que, en consecuencia, puede dar a nuestra presencia en el mundo, en ocasiones, un aspecto desapegado y un estar como un deambular sin dirección.

Si pensamos en cómo este panorama permea las discusiones educativas, nos encontraremos con que, junto con esta desafección de actitudes críticas, existe un paradójico entusiasmo, casi terapéutico, hacia el "pensamiento crítico". Así en este artículo, y como indica el título, en el primer movimiento, presentamos los elementos fundamentales de su tratamiento pedagógico contemporáneo. En el segundo movimiento, siguiendo la metáfora musical, proponemos una serie de variaciones en torno al pensamiento crítico. Finalmente, en el tercer movimiento, sugerimos que la mejor vía para desarrollar un pensamiento más auténticamente crítico es la transmisión de un contenido disciplinar rico en ideas e inserto en una tradición sujeta al diálogo y el debate continuos.

\section{PRIMER MOVIMIENTO: TRATAMIENTO PEDAGÓGICO CONTEMPORÁNEO DEL PENSAMIENTO CRÍTICO}

El análisis del pensamiento crítico no tiene nada de novedoso. Lo que sí lo tiene es la presencia de un extraordinario y renovado interés por los trabajos que abordan el tratamiento pedagógico del pensamiento crítico. Tanto, que puede 
hablarse de un «movimiento» de renovación pedagógica ${ }^{1}$, materializado, por un lado, en la proliferación de programas para enseñar a pensar (CTT, 2018; Muñoz Hueso, 2018; Bernardo, 2017; Swartz et al., 2013); y, por otro, en los discursos profesionalizantes acerca de la enseñanza y la transferencia de competencias en la educación formal (OECD, 2018; Ventura, Lai y DiCerbo, 2017; Howie, 2012)2.

En el origen de este renovado interés pedagógico por el pensamiento crítico, nos encontramos sin duda con la psicologización que han vivido los estudios sobre educación en las últimas décadas. Una disciplina, la psicología, que ha investigado las capacidades y funciones implicadas en el pensamiento crítico entendido como una estrategia cognitiva, y cuyos resultados habrían sido llevados a término en el ámbito escolar (Prieto, 2018; Martín y Barrientos, 2009). Este auge de las aplicaciones educativas de la psicología cognitiva tiene como punto de partida el influyente informe estadounidense $A$ Nation in Risk, que identificaba las deficiencias en los procesos de pensamiento de alto nivel (entre las que se cuenta el "pensamiento crítico") como la mayor debilidad del sistema educativo del país, siendo así que su enseñanza se convirtió desde entonces en una parte esencial del currículum escolar (Mehta, 2015) ${ }^{3}$

Si la psicología cognitiva está en el origen del renovado interés por el tratamiento pedagógico del pensamiento crítico, es muy posible que la encontremos

1. Un movimiento con sus correspondientes «olas» (PAUl y ELDER, 2005; PAUL, 1997) y que puede llegue a ser equiparable a los que recorrieron los sistemas educativos de medio mundo a principios del siglo xx. La vitalidad de este movimiento puede comprobarse, por ejemplo, en que dentro de unos meses se va celebrar en California la $38^{\text {th }}$ Annual International Conference on Critical Thinking. Ver también a este respecto: Mulnix (2013) y Difabio DE ANGLAT (2005).

2. Un discurso educativo centrado en la adquisición de competencias que está también presente en los niveles de educación primaria y secundaria. En el contexto español, el debate sobre el desarrollo del pensamiento crítico está de plena actualidad, por la preocupación que entre los políticos despiertan los efectos de las fake news (hace unos pocos meses la vicepresidenta del Gobierno afirmaba: "Tenemos entre todos que dotar a nuestros hijos de herramientas y conocimientos para desarrollar un pensamiento propio y crítico sobre los contenidos", EUROPA PREss, 2017) y por el debate sobre el lugar y horas asignadas a la materia de filosofía en las escuelas (Pérez LEDO, 2018; MARina, 2018; MORENO, 2017). En el contexto británico y estadounidense, existe una variedad amplia de maneras en las que de un modo directo o indirecto el pensamiento crítico se ha introducido en las prácticas escolares (NosicH, 2012; PETERS, 2007).

3. Es interesante que fuese el enfoque cognitivista de la psicología el que calase más ampliamente en la educación, pues, como es sabido, se caracteriza por estudiar los procesos mentales que participan en la generación y construcción del conocimiento, interesándose por cómo las personas entienden la realidad y cómo ello orienta y determina su acción. Las terapias psicológicas "cognitivoconductuales» que desarrolla suelen ser breves y están orientadas a la solución de problemas concretos por medio de protocolos de tratamiento muy estructurados. Una psicología que podríamos denominar "procedimental", donde el sujeto de la terapia más que ser «objeto de una elaboración [o reelaboración] ardua y compleja" (Foucault, 1984/1994, 10) lo es de intervenciones operativas y eficientes. También aquí los grandes relatos sobre el yo parecen agotados, y se impone un enfoque tecnológico y operativo del análisis de las problemáticas y la propuesta de soluciones que permitirán salir a ese yo, quizá con cierto cinismo y desafección hacia sí mismo, de las situaciones de crisis, como propone la mayoría de la literatura superventas de autoayuda seguidoras de la psicología positiva. 
también en su desarrollo contemporáneo. ¿Cómo se entiende educativamente hoy el pensamiento crítico?

En primer lugar, podemos decir que el pensamiento crítico es sinónimo de "pensar bien", siendo así un concepto normativo que implica una serie de habilidades y disposiciones. Sobre esto, parece existir un amplio consenso. Por ejemplo, Ennis, señalado en la actualidad como «uno de los fundadores del campo de investigación del pensamiento crítico» (Davies y Barnett, 2015, 27), lo identifica como «un tipo de pensamiento razonado y reflexivo que se centra en decidir qué creer y qué hacer» (Ennis, 2015b, 32; 1987, 10). Desde el campo de la filosofía de la educación, hace ya tiempo que, por ejemplo, Siegel viene manteniendo una perspectiva parecida al afirmar que el pensamiento crítico significaba estar «debidamente movido por razones» (2006, 23). En el contexto español, Ibáñez-Martín considera que la actividad intelectual está «llamada a alcanzar un auténtico saber, mediante un trabajo de interpretación y organización sistemática de los elementos que nuestro conocer proporciona, ponderando el valor de cualquier dato, argumentación, prueba o idea" (2017, 245, cursiva en el original). Richard Paul, una de las figuras más destacadas en la atención actual que recibe el pensamiento crítico ${ }^{4}$, considera que el pensamiento crítico «es el proceso de analizar y evaluar el pensamiento con el propósito de mejorarlo [...] [que] presupone el conocimiento de las estructuras más básicas del pensamiento (los elementos del pensamiento) y los estándares intelectuales más básicos del pensamiento (estándares intelectuales universales)» (Paul y Elder, 2005, 7). Todo lo cual supondría evaluar críticamente las creencias, las asunciones ocultas y las cosmovisiones en que se inserta.

Un segundo rasgo característico en el tratamiento pedagógico contemporáneo del pensamiento crítico no es tanto un consenso, sino, por el contrario, una discusión. Se trata del debate acerca de si el pensamiento crítico debe ser transmitido de modo general y transversal o si debe serlo a través de contenidos curriculares específicos. Los argumentos a favor de su enseñanza contextualizada por medio de materias específicas señalan que siempre pensamos sobre algo y que pensar bien acerca de algo implica atenerse a los criterios propios del ámbito de ese algo. Así se ha pronunciado siempre McPeck (2017), para quien la adquisición de competencias críticas solo puede tener lugar por la mediación de contenidos temáticos específicos; una perspectiva que se enmarcaría en el proyecto de la educación liberal, el cual tiene como principal enemigo, precisamente, a quienes consideran el aprender a pensar como un fin en sí mismo. Aquellos que, por el contrario, defienden un tratamiento generalista del pensamiento crítico insisten en el hecho de que contiene rasgos que son aplicables a distintos ámbitos, entre los que destacan: (i) el reconocimiento de argumentos falsos y contradicciones, así como la preocupación por la consistencia de los mismos; (ii) el rechazo del relativismo

4. Una revisión exhaustiva de las aportaciones realizadas por Richard Paul al análisis del pensamiento crítico puede encontrarse en el número homenaje que ELDER y Nosich (2016) han editado en la revista INQUIRY. La formulación inicial de sus ideas se encuentra en PAUL (1990). 
frente a la objetividad; (iii) la asunción de qué justificaciones racionales pueden ser falsas, en cuyo caso pueden no estar determinando la verdad; y (iv) el desarrollo de un "espíritu crítico». En este sentido, Bailin y Siegel (2003) rechazan cualquier tipo de jerarquización del pensamiento -en la línea de las clásicas taxonomías de Bloom (1972) o Ennis (1987)-, al considerar que es mejor pensar en los criterios del pensamiento crítico en términos de su aplicación, con independencia de la tarea y del contexto. Así, "de acuerdo con los defensores de esta concepción más amplia del sentido crítico la educación debería tener como finalidad fundamental el desarrollo en los estudiantes de (1) la habilidad de razonar bien [...] y (2) la disposición e inclinación a ser guiado por razones así evaluadas (Siegel, 2010, 142).

Un tercer aspecto que puede ayudarnos a entender cómo se aborda hoy el pensamiento crítico en el ámbito educativo es la discusión acerca de la vinculación o diferencia entre el pensamiento crítico y el pensamiento creativo. En ocasiones, el pensamiento crítico es visto como una especie de proceso algorítmico -con procedimientos paso a paso, rigurosamente organizados con respecto a unos criterios determinados-, mientras que el pensamiento creativo es visto como la acción de trascender los marcos establecidos y de crear algo nuevo, a través de la puesta en marcha de la imaginación. Desde esta perspectiva lo explicaba ya Lipman (1991, 254) cuando, a la hora de establecer las diferencias existentes entre el pensador creativo y el pensador crítico, señalaba sobre este último que era de naturaleza conservadora mientras que el primero se caracterizaba por ser más escéptico y radical. Algunos autores mantienen también la conveniencia de esta distinción basándose en criterios de formación inicial investigadora (Brodin, 2016, 974). Sin embargo, y en contra de dicotomías como esta, puede decirse que en el pensamiento crítico siempre participan la imaginación y la creatividad -en la identificación de los problemas, en la interpretación de una situación y a la hora de enfrentarse a situaciones nuevas-; y que, de modo similar, el pensamiento creativo implica también la evaluación crítica, el análisis y el juicio racionales. Así, la acción de pensar bien es un pensamiento, al mismo tiempo, crítico y creativo porque al ser "razonable y reflexivo, implica una apelación a las razones, implica juicio, es dialéctico y se adhiere a las normas, estándares y criterios, y es creativo ya que implica cuestionar suposiciones y descartes de creencias, generación de ideas, suministro de constructos imaginativos» (Bailin, 1993, 162).

Pero, ¿por qué el pensamiento crítico debería considerarse fundamental en educación? Pueden darse cuatro grandes razones para ello, las cuales son mayoritariamente asumidas a la hora de justificar la pertinencia e importancia de la educación del pensamiento crítico. Una justificación que entra en relación con una consideración de la dimensión ético-moral de la formación, no ya en determinadas "habilidades o competencias críticas", sino en la formación de "pensadores críticos". Estas razones, derivadas de los influyentes trabajos de Siegel (2017, 6-7; 2006, 55-61) en la materia, son las siguientes: (i) El principio del respeto a las personas requiere que las reconozcamos como capaces de pensar críticamente y que promovamos entre ellas esa capacidad. Los alumnos tienen derecho y capacidad de pensar, 
cuestionar, desafiar y buscar sus propias preguntas, respuestas y razones, por lo que habría que desarrollar una práctica educativa que tuviese en consideración el hecho de que los educandos son sujetos de esos derechos y que llegan a nuestras aulas con determinadas capacidades. (ii) El pensamiento crítico es necesario en la preparación para la vida adulta. Para pasar a formar parte con éxito de la comunidad de adultos, las nuevas generaciones tienen que ser capaces de elaborar sus juicios y opiniones, así como sus afectos y emociones, de manera independiente. (iii) El pensamiento crítico juega un papel central en las actividades intelectuales superiores (los contenidos de las disciplinas) que son una preocupación educativa por su papel en la capacitación para participar y contribuir en las tradiciones racionales y prácticas razonables ${ }^{5}$. La formación del pensamiento crítico no es solo un fin sí misma, sino que también es un medio que facilita la participación de los alumnos como miembros competentes de la comunidad de investigación crítica. (iv) El pensamiento crítico es esencial para la continuidad de la democracia y la formación cívica de los alumnos, pues es de esperar que un buen pensador crítico, sea un buen ciudadano, capacitado para participar y contribuir plena y conscientemente en la sociedad democrática en que vive.

\section{SEGUNDO MOVIMIENTO: VARIACIONES DEL PENSAMIENTO CRÍTICO}

En lo que sigue, vamos a tratar de explorar las maneras en las que el concepto de pensamiento crítico es aislado, en ocasiones, de la idea de crisis, pero que, sin embargo, está ahí, escondida, en el término "crítico» para, a continuación, tratar de establecer así una consideración más amplia y rica en matices del pensamiento crítico en el campo educativo de la que, como hemos tratado de resumir en el apartado anterior, viene ofreciéndose.

Existen cuatro grandes problemáticas por medio de las cuales se reduce y minimiza el componente «crisis» presente en el pensamiento crítico:

i. La variable «crisis» del pensamiento crítico se oculta, en primer lugar, tras la imagen que se ofrece del sujeto dotado de pensamiento crítico. Esta imagen es la de una persona autónoma, segura de su propia presencia y que controla la situación mientras evalúa el terreno en que se sitúa y las condiciones en que se produce el juicio. Nos parece que esto constituye un retrato falso del ser humano. El instrumento que representa la capacidad de discernimiento en la justicia, la balanza, no se caracteriza, sin embargo, por aportar ningún criterio claro de discernimiento que permita determinar la falsedad o verdad de determinada cosa o evaluar el valor de la cosa en

5. La incorporación del concepto de razonabilidad (reasonableness) al debate educativo-filosófico en torno al pensamiento crítico, como la disposición de aplicar la habilidad de razonamiento lógico a contextos de práctica concretas, es atribuible a Burbules (2016, 1995a, 1995b, 1992, 1991; BurBules y BERK, 1999). 
sí, sino que, al contrario, expresa los equilibrios y desequilibrios de la(s) cosa(s) sopesada(s). De modo similar, una persona que piensa, y que lo hace en las condiciones epistemológicas que han seguido a la crisis de la modernidad, toma más la forma de un caminar por pasos dubitativos, inseguros, que el de la figura que avanza, segura de su gravedad, con paso firme.

ii. Otra forma de reducir y minimizar el componente "crisis» presente en el pensamiento crítico opera a través del lenguaje. En efecto, en ocasiones, el lenguaje es tratado, en este contexto, como relativamente poco problemático, como si constituyese el fondo, más o menos estable, para el ejercicio de la racionalidad, que es donde la tarea del pensamiento crítico tiene lugar. La atención que la filosofía desde el siglo Xx viene prestando al lenguaje pone en evidencia que este se aleja mucho de no ser problemático en sí mismo. Además, estas atenciones se han dirigido precisamente a "poner en crisis» la idea misma del lenguaje como un ente mediador (totalmente interpretable, enteramente perfectible y absolutamente controlable) entre el «yo» y la "realidad», por lo que es difícilmente justificable, como así suele suceder, que el-problema-del-lenguaje o el-lenguaje-como-problema sean ignorados en la habitual caracterización del pensamiento crítico que se hace en el ámbito pedagógico.

iii. Otro posible reduccionismo proviene de que la imaginación es vista como un elemento que opera habitualmente junto con el análisis crítico, como un paso o componente natural en algunos puntos del proceso de dicho análisis crítico, para que así las cosas puedan desarrollarse de un modo creativo. Sin embargo, mientras que "crítico" y "creativo" pueden ser términos que hacen hincapié en diferentes aspectos del pensamiento crítico, creemos que estos deben interpretarse, estrictamente, como formas de pensar distintas. Decimos esto porque la creación de algo nuevo exige, muchas veces, evadirse de la realidad y las descripciones que sobre ella ya existen, saliendo de los esquemas convencionales para poder así crear otros distintos. Y evadirse de la realidad no es lo mismo que criticarla.

iv. Otra tendencia a minimizar el componente "crisis" del pensamiento crítico proviene de que los problemas teóricos y prácticos que surgen en la realidad y que provocan que las crisis no surjan espontáneamente de la nada son, en sí mismos, constitutivos de las tradiciones de pensamiento que operan en el modo habitual de entender la interacción del sujeto con su cultura. Dicho de otro modo, los problemas no emergen de un terreno ya de por sí llano, diáfano, transparente, sin complicaciones. Muy al contrario, si puede hablarse de una "tradición "crítica" en Occidente" (Foucault, 1983/2004, 213), es porque, precisamente, el encuentro del sujeto con la realidad es problemático de suyo, expresándose en diversas direcciones y sentidos, especialmente, porque siempre ha sido ya un problema, antes de su cuestionamiento, "Saber si un individuo particular dice la verdad" (ibid., 
41) acerca de algo. Un problema que se agrava y extiende, en nuestro contexto cultural, desde la introducción de la radical duda cartesiana de si "lo que crees es, de hecho, verdad" (idem).

A continuación nos vamos ocupar con más detalle del cuestionamiento de algunas de estas problemáticas que pueden estar reduciendo o minimizando el marco interpretativo del término «crisis» dentro de la conceptualización pedagógica dominante del pensamiento crítico $^{6}$. Y lo vamos hacer mostrando tres ejemplos que representan, a su vez, variaciones de modos de pensar que ponen en crisis la idea de crítica popularmente aceptada. Para mostrar esto, será necesario recurrir a ciertas formas del lenguaje filosófico y a modos de pensar que rompen o se distancian con el discurso y los términos habituales que en pedagogía empleamos para referirnos al pensamiento crítico. El objetivo de esta reconsideración filosófico-educativa radica, como dijimos al principio, en mejorar la consistencia pedagógica de los propósitos educativos habituales en las propuestas para mejorar el pensamiento crítico.

\subsection{Variación número uno: Stanley Cavell y el Walden de Thoreau}

En su relato sobre la época que pasó viviendo en Walden Pound, Thoreau (1854/2013) comparaba la condición humana a la de los animales que observaba en los bosques de alrededor. Veía un pájaro que estaba perdiendo las plumas y una serpiente que mudaba de piel, y describía a estos animales como seres en estado de crisis. Por su parte, la obra de Stanley Cavell (1972/2011) Los sentidos de Walden lleva como epígrafe las palabras de Martín Lutero: "Porque toda nuestra vida debe ser un bautismo». La aparición de la novedad no ha de interpretarse solo como una posibilidad contingente, algo que nos puede suceder, que aparece cuando menos nos lo esperamos, fuera de nuestras expectativas, sino también como algo que es, en cierto modo, responsabilidad nuestra. Esto es especialmente así en relación con las palabras que empleamos para nombrar el mundo, es decir, las palabras que utilizamos para configurar el terreno en el que nos encontramos y con las que, del mismo modo, nos configuramos en el modo de estar y mirar ese mundo. Un terreno que nunca es llano, que nunca es conocido del todo; un terreno desde el que, gracias precisamente a la presencia de sus desniveles, aparecen no solo nuestras respuestas, sino aun nuestra misma capacidad de respuesta. Un terreno que, además, está siempre abierto a la posibilidad de verse dividido, quebrado.

En su comentario sobre Walden, Cavell (1972/2011) muestra los modos en que, por sí mismo, el proceso de lectura nos enfrenta constantemente con puntos de inflexión y con la toma de decisiones, sobre todo, cuando se trata de textos

6. Nótese, en la relación entre "crítica» y "crisis", la conexión etimológica entre ambos conceptos:

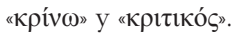


complicados como el de Thoreau (1854/2013). Si leemos correctamente, nos encontramos continuamente divididos, quebrados, dubitativos, inseguros sobre cómo entender una expresión o una oración ante cuyas afirmaciones o negaciones nos situamos, y que nos instala en relación con el texto (y aun con nuestras vidas) como un todo. $\mathrm{Y}$ es en esos momentos de decisión, donde existe esa posibilidad de la novedad que es una especie de "bautismo".

Pues bien, es importante reconocer que los momentos de decisión aquí anunciados, las dudas permanentes, las experiencias de las continuas crisis, las incertidumbres para elegir entre dos o más opciones, no se entiendan en los mismos términos que el tipo de toma de decisiones presente en las descripciones analíticas habituales del pensamiento crítico. No se trata, en aquellos casos, de tomar distancia para, a continuación, considerar racionalmente las ventajas y desventajas, los pros y los contras, de una situación particular. Estos momentos están más bien entretejidos, entremezclados, en la experiencia de la lectura en sí, y es esta experiencia la que debe, precisamente, tomarse como indicativa de lo que realmente son nuestras vidas entendidas como un todo. Este tipo particular de experiencia lectora al que hemos hecho referencia y que sustancializa lo que ocurre en nuestras vidas no es algo que construimos de forma calculada, medida, secuencializada, sino que, más bien, se convierte en elemento característico de la forma en que vive una persona educada.

Lo que hemos tratado de mostrar aquí es una primera variación posible sobre el modo habitual de pensar el elemento 'crisis' dentro de la enseñanza del pensamiento crítico, consistente en reconocer que nuestras vidas se encuentran permanentemente entreveradas de tomas de decisión, de dudas, de incertidumbres, frente a las cuales no hay una forma sistematizada o protocolizada de pensar críticamente. Y esto es así porque, en rigor, esos son los contornos, los desniveles, las novedades, en fin, las condiciones previas que hacen posible, precisamente, el ejercicio del pensamiento crítico tal y como es comúnmente entendido en pedagogía. De este modo, una persona educada en esta variante previa del pensamiento crítico se caracterizaría más por saber aceptar-se en lo azaroso, que por saber cuestionar y cuestionar-se críticamente en aquello que hay de previsible.

\subsection{Variación número dos: John Llewelyn y la imaginación}

En su trabajo La Imaginación HipoCrítica, John Llewelyn (2000) explora una serie de ideas interesantes para el tema que nos ocupa, y lo hacía en los siguientes términos. La hipocresía es comúnmente considerada como una falta personal grave: consiste en, por ejemplo, decir una cosa y hacer o creer otra distinta, y (generalmente) además, hacerlo sin sinceridad. Se trata de estar ante un dilema, entre la espada y la pared, dividido. Pero Lévinas, llamando la atención sobre una implicación más sutil de esta expresión, sugería que: "Tal vez sea tiempo de reconocer en la hipocresía, no sólo un despreciable defecto contingente del hombre, sino el desgarramiento profundo de un mundo ligado a la vez a los filósofos y a 
los profetas» (Lévinas, 1971/2002, 50). Los "filósofos» apoyarían aquí a ese tipo de objetividad que supone un reconocimiento totalizador del terreno, el espacio en el que se ejerce el pensamiento crítico. Los "profetas», en cambio, apostarían por una orientación siempre abierta a nuevas posibilidades. La primera postura, la filosófica, parece adecuada para un objetivismo plano, mientras que la segunda, la profética, sería necesaria para un tipo de perfectibilidad más abierta. Por lo tanto, lo primero puede asociarse con lo real, pero lo segundo con lo posible, y es importante recordar que los seres humanos en situación educativa no viven solo en el reino de lo real. Así, el predominio de lo primero esconde las formas por las cuales siempre hay un desagarro en la vida humana -que el mundo mismo debe entenderse siempre en términos de este desgarro que se abre entre la realidad objetiva y todo lo que es posible-. Dicho de otra manera, el mundo nunca es la contrapartida natural de los instintos humanos, de la manera en que lo es en el hábitat de los animales que viven en él. La realidad humana, cuando es pedagógicamente pensada, tiene tanto más que ver con las posibilidades que con sus condiciones objetivas de partida.

Llewelyn se esfuerza en demostrar que en nuestra comprensión del mundo siempre nos encontramos, antes que con la acción del pensamiento crítico, con la actividad de imaginar lo posible. Hay un ver como, y esto aparece en el nombrar de las cosas, en la enunciación del mundo. La conocida formulación del problema geométrico («Sea $a$ un ángulo de 85 grados...»), de hecho, demuestra algo que está, por así decirlo, escrito en nuestro entendimiento común y objetivo del mundo. Nos tomamos las cosas como tal o cual cosa. Se puede decir que el pensamiento crítico opera así en el nivel de razonamiento, donde el razonamiento es entendido en términos del análisis lógico y la administración de las proposiciones.

Ahora bien, del mismo modo que ocurría en el apartado anterior, con esta segunda variación en el modo de pensar la aparición del elemento "crisis» del pensamiento crítico, lo que queremos sugerir es que aquello que nos va a permitir poder empezar a ver los problemas y a pensar críticamente no es la aplicación mecánica de un proceso construido y cerrado de pensamiento, sino, precisamente, la aplicación de la imaginación -que no tiene límites- como una resistencia de ocurrencias que pretende o bien tambalear un estado evidente de cosas, o bien crear un mundo de posibilidades todavía no existente que al operar en un ejercicio de crítica no evade la realidad.

\subsection{Variación número tres: Gilles Deleuze y la creación de problemas}

¿Cuál es la consecuencia de unir el sustantivo «problemas» con el verbo «solucionar»? Pues que, a veces, ello fomenta la adopción de un enfoque técnico o cuasitécnico, y refuerza la hipótesis de que los problemas han de entenderse en estos términos. Esto oculta también la idea wittgensteiniana de que los problemas a veces no necesitan ser resueltos, sino disueltos. También oculta una especie de metafísica de la estabilidad, en la que las perturbaciones deben superarse 
continuamente en una especie de ejercicio homeostático constante, de búsqueda del equilibrio perfecto, de la armonía silenciosa.

En lugar de resolver problemas, Deleuze lo que nos propone es crearlos y, sobre todo, reconocer la relevancia que tienen en sí mismos, esto es, que el conocimiento está en la propia enunciación de los problemas y no solo en su solución. "Se nos hace creer que la actividad del pensar, y también lo verdadero y lo falso, con relación a esa actividad solo comienza con la búsqueda de soluciones, no concierne más que a las soluciones» (Deleuze, 1968/2002, 242). Parecería, pues, que lo que da el sentido a los problemas es la propuesta de soluciones y, por tanto, lo importante es su desaparición. "Por ello también se cree tan a menudo que la solución no deja subsistir al problema, y le da retrospectivamente el estatuto del momento subjetivo necesariamente superado cuando se encuentra la solución. Sin embargo, es completamente al contrario» (Deleuze, 1969/2005, 155).

Otro aspecto interesante de la propuesta de relación de Deleuze entre "problemas" y "soluciones» proviene de su idea del sentido con respecto al lenguaje. Normalmente se cree que el lenguaje (al menos en sus aspectos más centrales) funciona a través de una correspondencia entre las afirmaciones y los estados de las cosas -por ejemplo, "la botella está sobre la mesa" y el hecho de que la botella está sobre la mesa-. Pero Deleuze nos ayuda a ver cómo esto ignora otro aspecto muy importante del lenguaje: el sentido que tienen para nosotros. Nuestra enunciación del mundo no es separable del mundo, no en la forma en que la imagen de un espejo se relaciona con el objeto que refleja, sino que pone nuevos elementos en el mundo -como bien saben los publicistas- y, en ese mismo proceso, extiende el propio mundo, el significado, el sentido de las palabras.

La palabra "sentido" en francés (sens) contiene la idea tanto de sentido como de dirección, es decir, de movimiento (un sens unique es una calle de sentido único). Pues bien, en el modo en que enunciamos los problemas enunciamos el sentido de la pregunta que los ha abierto y, esto es ahora lo relevante, ese sentido permanece sea cual sea la solución propuesta: «El problema en sí mismo es la realidad del elemento genético, el tema complejo que no se deja reducir a ninguna tesis de proposición" (Deleuze, 1969/2005, 155). De este modo, los problemas no desaparecen al resolverlos, pero tampoco están hechos o enunciados claramente desde el principio, sino que hay que crearlos, elaborarlos, y en ese mismo proceso hacemos que persista y permanezca el sentido o tema que los constituyó. La fuerza del conocimiento, pues, se reconoce en que los problemas no son, en sí mismos, meras carencias, o privaciones, o dificultades, que solo apuntan y merecen identificarse en las soluciones que les proponemos, sino en la entidad objetiva del tema que les sostiene.

Con esta tercera variación en el modo de pensar el elemento "crisis» dentro del pensamiento crítico, tratamos de llamar la atención sobre la importancia que tiene no solo resolver el problema, o disolverlo, o trascenderlo, sino saber reconocer también en él, en su planteamiento, en su formulación o definición, la fuerza del conocimiento. De este modo, cabe considerar la importancia que tiene, dentro de 
la proyección educativa del pensamiento crítico, no solo la búsqueda obsesiva, eficiente y eficaz de soluciones, bien teóricas o prácticas, sino también saber reconocer la identidad esencialmente crítica que tiene en sí misma la existencia de los problemas, su creación y su elaboración, así como el tema y sentido del cual y por el cual surgieron.

\section{TERCER MOVIMIENTO: CIERRE Y CONCLUSIONES}

En nuestra opinión, el tratamiento que el pensamiento crítico recibe habitualmente en el ámbito educativo, especialmente desde los campos más psicológicos y didácticos, acentúa erróneamente, como se ha mostrado, una serie de supuestos. Entre estos nos hemos aproximado a los siguientes tres: primero, que la transmisión de la imagen del pensar crítico es un pensar desencarnado, cuando el pensar y el vivir son un proceso, así lo mostramos, desde el ejemplo de la lectura, entreverado, entremezclado, en continuas tomas de decisiones, de dudas, de incertidumbres que carecen de un sistema de pensamiento crítico estandarizado, pero que, en cualquier caso, establecen las condiciones previas que harán posible el pensamiento crítico. Segundo, que siendo la imaginación uno de los elementos esenciales del pensar crítico no es, sin embargo, susceptible de ser aplicada desde un proceso mecánico de análisis. Y, tercero, que el elemento crítico del pensamiento no ha de focalizarse solo en la búsqueda de soluciones, sino en la capacidad de dar entidad a un problema y, por tanto, a un nuevo posible tema y sentido.

Estas condiciones no pueden regularse o construirse estandarizamente pues finalmente desconocemos la conectividad o vinculación que desencadena el pensar, en sí mismo crítico, imaginativo y generador de ideas. Lo que sí sabemos es que si realmente queremos que los educandos vivan y se aproximen a las condiciones señaladas, es necesario promover el compromiso del educador con un contenido de aprendizaje temáticamente rico. Esto se ve más claramente cuando los estudiantes se inician en modalidades de investigación (materias, áreas curriculares, asignaturas) a las cuales preceda una larga tradición. La tradición aquí debe entenderse no como la transmisión de un corpus fijo de creencias, sino más bien con arreglo a los siguientes cuatro factores: (i) debe contener prácticas sostenidas de lectura, donde estas impliquen determinados estilos de argumentación y enfoques y contengan referencias continuadas (por ejemplo, a otros textos); (ii) debe ser digna de ese nombre al incorporar la crítica en su práctica, y esta debe evolucionar; (iii) debe contar con sus bandos, sus cambios de paradigma, sus disputas rivales; y (iv) debe contener su propia vanguardia. El objetivo es, pues, una iniciación en esta crítica práctica a través de una confrontación con (una muestra representativa de) el contenido que define la práctica objeto de estudio. Las implicaciones pedagógicas que esto tiene para pensar los contenidos de enseñanza, los medios para su transmisión y la orientación que la formación del profesorado debiera tomar son importantes: resituar en el centro de la tarea educativa el contenido y su tradición; rediseñar las situaciones didácticas como ejercicios de iniciación en la tradición 
heredada; preparar a los docentes para ser guardianes de dicha tradición capaces de preparar a sus alumnos para su transformación.

Un compromiso por esta vía es quizá menos cómodo, pero evita que caigamos en la falsa confianza y autocomplacencia de creer que el desarrollo del pensamiento crítico puede alcanzarse exclusivamente por procedimientos técnicos. Como hemos visto, los "procedimientos" y las "técnicas" para sopesar racional y críticamente los elementos de la realidad tienden a alejar, a distanciar, a los sujetos de esa realidad, de la instancia de que se trate. Cuando, en ocasiones, lo que hay que hacer es, precisamente, lo contrario, acercarnos, aproximarnos, mezclarnos, tanto a ella como para dejarnos afectar por lo que pasa a nuestro alrededor, por lo que sucede frente a nuestros ojos, por lo que pensamos y sentimos, por todo aquello que leemos, vemos y escuchamos.

\section{REFERENCIAS BIBLIOGRÁFICAS}

BAILIN, S. (1993) Epilogue: Problems in Conceptualizing Good Thinking. American Behavioral Scientist, 37 (1), 156-164.

Bailin, S. y Siegel, H. (2003) Critical Thinking, en Blake, N.; Smeyers, P.; Smith, R. y STANDISH, P. (eds.) The Blackwell Guide to Philosophy of Education. Oxford, Blackwell, 181-193.

Bárcena, F. (2006) Hannah Arendt: una filosofía de la natalidad. Barcelona, Herder.

BERNARDO, J. (2017) El libro del educador: cómo enseñar a aprender y a pensar. Madrid, Rialp.

Bloom, B. J. (1972) Taxonomía de los objetivos de la educación: la clasificación de las metas educacionales. Buenos Aires, El Ateneo.

BRodin, E. M. (2016) Critical and creative thinking nexus: learning experiences of doctoral students. Studies in Higher Education, 41 (6), 971-989. https://doi.org/10.1080/03075 079.2014 .943656

BuRBules, N. C. (1991) Rationality and Reasonableness: A Discussion of Harvey Siegel's Relativism Refuted and Educating Reason. Educational Theory, 41 (2), 235-252.

Burbules, N. C. (1995) Reasonable Doubt: Toward a Postmodern Defense of Reason as an Educational Aim, en KoHLI, W. (ed.) Critical Conversations in Philosophy of Education. London, Routledge, 82-102.

Burbules, N. C. (2016) Being Critical About Being Critical. Democracy \& Education, 24 (2), 1-5. Consultado el 30 de abril de 2018. https://democracyeducationjournal.org/cgi/ viewcontent.cgi?referer=https://www.google.es/\&httpsredir=1\&article=1302\&context= home.

Burbules, N. C. y Berk, R. (1999) Critical Thinking and Critical Pedagogy: Relations, Differences, and Limits, en Popkewitz, T. S. y Fendler, L. (eds.) Critical Theories in Education: Changing Terrains of Knowledge and Politics. London, Routledge, 45-66.

Cavell, S. (1972/2011) Los sentidos de Walden. Valencia, Pre-Textos. [Traducción de Antonio Lastra].

Center for Teaching Thinking (CTT) (2018) Lessons. Consultado el 30 de abril de 2018. http://teach-think.org/es/recursos/lecciones-y-articulos/.

Davis, M. y BARnetT, R. (2015) The Palgrave Handbook of Critical Thinking in Higher Education. New York, Palgrave MacMillan. 
EL PENSAMIENTO CRÍTICO EN CRISIS. UNA RECONSIDERACIÓN PEDAGÓGICA EN TRES MOVIMIENTOS

Deleuze, G. (1968/2002) Diferencia y repetición. Buenos Aires, Amorrortu. [Traducción de María S. Delpy y Hugo Beccacece].

Deleuze, G. (1969/2005) Lógica del sentido. Barcelona, Paidós. [Traducción de Miguel Morey].

Difabio DE ANGLAT, H. (2005) El critical thinking movement y la educación intelectual. Estudios sobre Educación, 9, 167-187. Consultado el 30 de abril de 2018. http://hdl.handle. net/10171/8919.

Elder, L. y Nosich, G. (eds.) (2016) Memorial Issue to Richard Paul. INQUIRY. Critical Thinking Across the Disciplines, 31 (1), spring.

EnNIS, R. (1987) A Taxonomy of Critical Thinking Dispositions and Abilities, en BoykoffBaron, J. y Sternberg, R. J. (eds.) Teaching Thinking Skills: Theory and Practice. Oxford, Blakwell, 9-26.

EnNIS, R. (2015) Critical Thinking: A Streamlined Conception, en Davis, M. y BarnetT, R. (2015) The Palgrave Handbook of critical thinking in Higher Education. New York, Palgrave MacMillan, 31-47.

Europa Press (21 de noviembre de 2017) Saénz de Santamaría avisa del impacto de las 'fake news' en las opiniones y pide a los medios contrastar la información. Europa Press. Consultado el 30 de abril de 2018. http://www.europapress.es/sociedad/noticiasaenz-santamaria-avisa-impacto-fake-news-opiniones-pide-medios-contrastar-informacion-20171121111222.html.

Foucault, M. (1983/2004) Discurso y Verdad en la Antigua Grecia. Buenos Aires, Paidós. [Traducción de Fernando Fuentes].

Foucault, M. (1984/1994) ¿Qué es la Ilustración? Actual, 28, 1-18. [Traducción de Jorge Dávila]. Consultado el 30 de abril de 2018. http://www.saber.ula.ve/bitstream/ handle/123456789/15889/davila-que-es-la-ilustracion.pdf;jsessionid=15F9B4D8D4CDE 7688AEC434968209FCC?sequence $=1$.

HowIE, D. (2012) La enseñanza del pensamiento en la escuela: competencias de la educación cognitiva. Madrid, Popular.

IBÁÑ̃z-MarTín, J. A. (2017) Horizontes para los educadores. Las profesiones educativas y la promoción de la plenitud humana. Madrid, Dykinson.

LÉvinas, E. (1971/2002) Totalidad e infinito. Ensayo sobre la exterioridad. Salamanca, Sígueme. [Traducción de Daniel E. Guillot].

LIPMAn, M. (1991) Thinking in Education. Cambridge, Cambridge University Press.

Llewelyn, J. (2000) The HypoCritical Imagination: Between Kant and Levinas. Cambridge, Cambridge University Press.

Marina, J. A. (20 de febrero de 2018) El pensamiento crítico. El Confidencial. Consultado el 30 de abril de 2018. https://www.elconfidencial.com/alma-corazon-vida/ educacion/2018-02-20/el-pensamiento-critico_1524397/.

Martín, A. V. y Barrientos, Ó. (2009) Los dominios del pensamiento crítico: Una lectura desde la teoría de la educación. Teoría de la Educación, 21 (2), 19-44. Consultado el 30 de abril de 2018. http://revistas.usal.es/index.php/1130-3743/article/view/7150.

McPeck, J. E. (2017). Teaching critical Thinking. Dialogue and Dialectic. Abingdon, Routledge. Presentación: Michael Scriven.

мeнta, J. (2015) Escaping the shadow. A Nation at Risk and its far-reaching influence. American Educator, summer, 20-26. Consultado el 30 de abril de 2018. https://www.aft. org/sites/default/files/ae_summer2015mehta.pdf. 
Moreno, M. (24 de septiembre de 2017). Hace falta que el pensamiento crítico vuelva a las aulas. Diario ABC. Consultado el 30 de abril de 2018. http://www.abc.es/espana/castilla-la-mancha/abci-hace-falta-pensamiento-critico-vuelva-aulas-201709231750_noticia. html.

MulniX, J. W. (2013) Thinking critically about critical thinking. Educational Philosophy and Theory, 44 (5), 464-479. https://doi.org/10.1111/j.1469-5812.2010.00673.x.

MuÑoz Hueso, A. C. (2018) Enseñar a pensar: cómo favorecer el pensamiento crítico en el aula. Madrid, cCs.

Nosich, G. M. (2012) Learning to think things through: A guide to critical thinking across the curriculum. Boston, Pearson.

OECD (2018) Preparing our youth for an inclusive and sustainable world. The OECD PISA global competence framework. Paris, OECD. Consultado el 30 de abril de 2018. https://www. oecd.org/education/Global-competency-for-an-inclusive-world.pdf.

Paul, R. (1990) Critical Thinking: What Every Person Needs to Survive in a Rapidly Changing World. Rohnert Park, Center for Critical Thinking and Moral Critique.

Paul, R. (1997) The Critical Thinking Movement: 3 Waves. Foundation for Critical Thinking. Consultado el 30 de abril de 2018. http://www.criticalthinking.org/pages/ critical-thinking-movement-3-waves/856.

PAUl, R. y ElDER, L. (2014) Critical Thinking: Intellectual Standards Essential to Reasoning Well Within Every Domain of Human Thought, Part 4. Journal of Developmental Education, 37 (3), 34-35. Consultado el 30 de abril de 2018. https://files.eric.ed.gov/fulltext/ EJ1067273.pdf.

PAUl, R. y ELDER, L. (2005) Una guía para los educadores en los estándares de competencia para el pensamiento crítico. Fundación para el Pensamiento Crítico. Consultado el 30 de abril de 2018. https://www.criticalthinking.org/resources/PDF/SP-Comp_Standards. pdf.

PÉREZ LEDO, J. A. (27 de marzo de 2018) ¿Convendría una asignatura de pensamiento crítico? Eldiario.es. Consultado el 30 de abril de 2018. https://www.eldiario.es/zonacritica/ Convendria-asignatura-pensamiento-critico_6_754584538.html.

Peters, M. A. (2007) Kinds of Thinking. Styles of Reasoning. Educational Philosophy and Theory, 39 (4), 350-363. https://doi.org/10.1111/j.1469-5812.2007.00344.x.

PRIETO, M. (2018) La psicologización de la educación: Implicaciones pedagógicas de la inteligencia emocional y la psicología positiva. Educación XXI, 21 (1), 303-320. https://doi. org/10.5944/educXX1.16058.

Siegel, H. (2006) Educating Reason. Rationality, Critical Thinking and Education. London, Routledge.

Siegel, H. (2010) Critical thinking, en Peterson, P.; BAKer, E. y MCGaw, B. (eds.) International Encyclopedia of Education. New York, Elsevier, 141-145.

SIEGEL, H. (2017) Education's Epistemology: Rationality, Diversity, and Critical Thinking. Oxford University Press.

SloterdijK, P. (2007) Crítica de la razón cínica. Madrid, Siruela.

Swartz, R. J.; Costa, A. L.; Beyer, B. K.; Reagan, R. y Kallick, B. (2013) El aprendizaje basado en el pensamiento. Cómo desarrollar en los alumnos las competencias del siglo XXI. Madrid, SM.

Thoreau, H. (1854/2017) Walden. Madrid, Errata Naturae. [Traducción de Marcos Nava].

Ventura, M.; Lai, E. y Dicerbo, K. (2017) Skills for Today: What We Know about Teaching and Assessing Critical Thinking. London, Pearson. 